\title{
Trial-two goal arm alternation to orientation of trial-one starting stem
}

WILLIAM N. DEMBER, MICHAEL F. SHERRICK AND RAYMOND P. HARRIS, JR. UNIVERSITY OF CINCINNATI

Post-hoc analysis of data from a previously reported experiment revealed a tendency for rats to choose, on trial 2, that goal arm of a T-maze allowing movement in a direction opposite that afforded by the orientation of the starting stem of a different maze used on trial 1. This surprising effect, which was also obtained in a second, somewhat different, experiment, extends Douglas's finding of direction of movement as a cue for spontaneous alternation behavior, but also poses a problem for the procedure Douglas developed for neutralizing that cue.

In a thorough investigation of the stimuli which might serve as cues for spontaneous alternation behavior (SAB) in the rat, Douglas (1964) was able to identify only two such cues, one intra-maze and the other extramaze. The single intra-maze cue was the odor trail left by the rat on the maze floor during its first trial in the $\mathrm{T}$-maze. The single extra-maze cue was one related to direction of movement.

These two cues contribute to probability of SAB as independent sources, and, according to Douglas, they can be investigated in isolation. To eliminate odor trail as a cue to $S A B$, Douglas made use of paper floors which could be changed between trials. To eliminate direction of movement, Douglas employed either of two procedures: (a) two mazes are used, one for each of the two trials that are typically run in studies of SAB; the two mazes may be in different rooms, or in the same room, but in either case they are oriented perpendicular to each other; (b) one maze is used and is rotated $90^{\circ}$ between trials. By setting the goal arms of the trial-1 and trial-2 mazes orthogonal to each other, one assures that the tendency to alternate direction of the goal arm entered on trial 1 cannot be realized in the goal arms of trial 2. For example, suppose the goal arms on trial 1 point West and East; the rat entering the West arm emerges from that trial with a tendency to move in an Easterly direction. However, that tendency cannot affect his choice of goal arm on trial 2 if the alternatives available are goal arms pointing North and South.

The purpose of this paper is to report some evidence that casts doubt on the possibility of entirely eliminating the direction of movement cue through use of the Douglas procedure. This evidence points to the hitherto unsuspected influence on trial-2 goal-arm choice of the orientation of the starting stem on trial 1. Data from two experiments are reported below. The first experiment, which was designed for another, but related purpose (Sherrick \& Dember, 1966), revealed the effect in question via a post-hoc examination; the second experiment, also designed for another purpose, provides independent confirmation of the effect, with rats of a different strain and run under different circumstances.

Method

Experiment 1. The procedural details of the experiment have already been reported (Sherrick \& Dember, 1966). Of relevance here are the two conditions labeled $B$ and $C$ in the previous report. In condition $B$, the $T$-maze on trial 1 was oriented so as to enforce movement in the starting stem in a Westerly direction; in condition $\mathrm{C}$, movement in the trial-1 stem was in an Easterly direction. Rats from both conditions were given trial 2 in a maze oriented with its stem pointing South and goal arms allowing a choice between moving West or moving East. Data on trial-2 goal-arm choice are available for 18 of the 20 rats run in condition $B$, and for 19 of 20 in $\mathrm{C}$.

Experiment 2. Two identical mazes were used, painted flat black throughout. Starting stem and goal arm were 16 in. long, $4.5 \mathrm{in}$. wide, and $6.5 \mathrm{in}$. in height. Ss were 52 gentled male albino rats of the SpragueDawley strain. Each $S$ ran in a total of six conditions, in three of which the opportunity to respond to odor trail was available, but no evidence of SAB to odor trail was forthcoming. There was clear evidence of $\mathrm{SAB}$ to the directional cue available in the other three conditions, at a rate $(75 \%)$ about the same as that reported by Douglas. It was the three conditions originally intended as means of assessing reponse to odor trail that inadvertently provided evidence, similar to that obtained from Experiment 1, on the influence of trial-1 stem orientation on trial-2 goal-arm choice. That is, in these conditions the mazes for trial 1 and 2 were perpendicular to each other, and since odor trial manipulations were ineffective the original three conditions collapse to one, with two variants as described below. The stem of the trial-1 maze always enforced movement in a Northerly direction. The maze for trial 2 was located either to the right or to the left of the trial-1 maze, with goal arms in a North-South orientation, as shown in Fig. 1. Note that with the trial-2 maze on the left, a right turn would take $S$ in a Southerly direction (opposite to the direction of movement in the trial-1 maze stem), while with the trial-2 maze on the right a left turn was necessary to take the $S$ in a Southerly direction. Each of the 52 Ss ran once (i.e., 


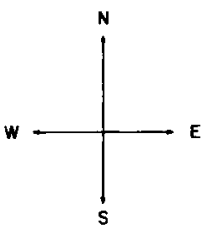

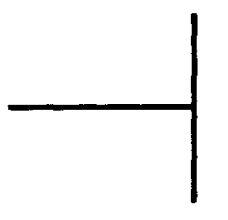

TRIAL 2

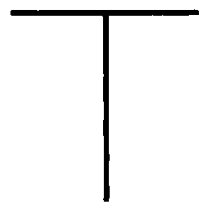

TRIAL I

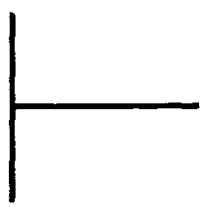

TRIAL 2
Fig. 1. Maze orientations for Experiment 2.

two trials, $30 \mathrm{sec}$. apart) in one of these configurations and twice in the other, generating a total of 156 test trials.

Results

Experiment 1. In condition B, 11 of $18 \mathrm{Ss}(61 \%)$ turned into the East goal arm on trial 2, and 13 of 19 $(68 \%)$ turned into the West arm in condition C. Combining the data from the two conditions, 24 of $37 \mathrm{Ss}$ (65\%) alternated between trial-1 stem direction and direction of trial 2 goal arm. By a $\chi^{2}$ test, the combined results differ from a chance distribution at a significance level between .10 and .05 ( $\left.\chi^{2}=3.26\right)$. Experiment 2. Alternation between trial-1 stem direction and direction of goal arm entry on trial 2 occurred in 93 of the 156 test trials $(60 \%)$. By a $\chi^{2}$ test this result differs from a chance distribution at a significance level between .02 and $.01 \quad\left(\chi^{2}=5.77\right)$. Discussion

There are obvious criticisms of these results. Neither experiment was designed for the purpose to which it has been put, and the cell entries for the analysis were not independent. There is also the possibility thai Experiment 2 merely reveals a South-going preference, though when this was checked by giving Ss only one trial in either the left- or right-hand maze, if anything there was a slight North-going preference.

The solution to these problems is also obvious: a replication experiment, designed to test properly the hypothesis in question. In the meantime, the present results seem worth calling to the attention of others who may be working on $\mathrm{SAB}$ within the Douglas framework. To be especially emphasized is the strong possibility that the Douglas procedure for neutralizing direction of movement as cue for SAB may do so for the goal arm of the trial-1 maze, but in so doing may endow significance to the orientation of the trial-1 starting stem. If our present results prove reliable, then Douglas's procedure may not provide a perfect means of investigating the odor trail cue entirely in isolation.

\section{References}

Douglas, R. J. An analysis of spontaneous alternation cues. Unpublished doctoral dissertation, University of Michigan, 1964. Sherrick, M. F., \& Dember, W. N. The tendency to alternate direction of movement as reflected in starting stem running speed. Psychon. Sci., 1966, 6, 29-30. 